\title{
Study Biocompatibility and Osteogenic Differentiation Potential of Human Umbilical Cord Mesenchymal Stem Cells (hUCMSCs) with Gelatin Solvent
}

\author{
Nike Hendrijantini ${ }^{*}$, Utari Kresnoadi1, Sherman Salim1, Bambang Agustono1, \\ Endang Retnowati², Iwan Syahrial ${ }^{3}$, Pungky Mulawardhana4, Manggala Pasca Wardhana4, \\ Coen Pramono5, Fedik Abdul Rantam ${ }^{6,7}$ \\ ${ }^{1}$ Department of Prosthodontic, Faculty of Dental Medicine, Airlangga University, Surabaya, Indonesia \\ ${ }^{2}$ Department of Clinical Pathology, Airlangga University \& Dr. Soetomo General Hospital, Surabaya, Indonesia \\ ${ }^{3}$ Department of Pathology, Faculty of Veterinary Medicine, Airlangga University, Surabaya, Indonesia \\ ${ }^{4}$ Department of Obstetric \& Gynecology, Airlangga University \& Dr. Soetomo General Hospital, \\ Airlangga University, Surabaya, Indonesia \\ ${ }^{5}$ Department of Oral and Maxillofacial Surgery/Dental Hospital, Faculty of Dental Medicine, \\ Airlangga University \& Dental Hospital, Surabaya, Indonesia \\ ${ }^{6}$ Laboratory of Stem Cell, Institute of Tropical Disease, Airlangga University, Surabaya, Indonesia \\ ${ }^{7}$ Regenerative Medicine \& Stem Cell Centre, Airlangga University \& Dr. Soetomo General Hospital, Surabaya, \\ Indonesia \\ Email: ${ }^{*}$ hendrijantininike@yahoo.com
}

Received 13 May 2015; accepted 5 July 2015; published 9 July 2015

Copyright (C) 2015 by authors and Scientific Research Publishing Inc.

This work is licensed under the Creative Commons Attribution International License (CC BY). http://creativecommons.org/licenses/by/4.0/

(c) (i) Open Access

\section{Abstract}

The human umbilical cord is a source of numerous Mesenchymal Stem Cells (MSCs), making it as a potential source of allogeneic multipotent cell for bone tissue engineering. The aims of this study were to find: 1) Human Umbilical Cord Mesenchymal Stem Cells (hUCMSCs) phenotypic characterization, 2) The in-vitro osteogenic differentiation potential of hUCMSCs, 3) The cytotoxicity of gelatin solvent to hUCMSCs using 3-[4,5-dimethylthiazol-2-yl]-2,5-diphenyl tetrazolium bromide (MTT) assay. As a result, through characterization of hUCMSCs, the majority of target cells expressed specific MSCs markers, Cellular Differentiation (CD)73, smaller number of subpopulation expressed CD90 with only minimal subpopulation expressed CD105 and all negative MSCs markers. Osteoblastic differentiation was found in a significantly high number of cells when in vitro osteogenic differentiation of hUCMSCs with Alizarin Red staining was done. Biocompatibility analysis using the MTT assay showed that gelatin solvent and Alpha modification of minimum essential

\footnotetext{
${ }^{*}$ Corresponding author.
}

How to cite this paper: Hendrijantini, N., et al. (2015) Study Biocompatibility and Osteogenic Differentiation Potential of Human Umbilical Cord Mesenchymal Stem Cells (hUCMSCs) with Gelatin Solvent. J. Biomedical Science and Engineering, 8, 420-428. http://dx.doi.org/10.4236/ibise.2015.87039 
medium Eagle ( $\alpha$-MEM) was non-toxic for hUCMSCs in vitro. The study concluded that hUCMSCs isolated from human umbilical cord was capable of undergoing in vitro osteogenesis, indicating its potential as allogeneic stem cells for clinical application in bone tissue engineering.

\title{
Keywords
}

\author{
Human Umbilical Cord Mesenchymal Stem Cells, Mesenchymal Stem Cells, Phenotypic \\ Characterization, Osteogenic Differentiation, Bone Tissue Engineering
}

\section{Introduction}

Bone tissue engineering can be done by giving osteoprogenitor cells [1]. The advanced development of cell therapy has brought about the possibilities to conduct such an ideal bone regeneration method using multipotent cells. Due to their abilities to self-renew and differentiate into various tissues like bone tissue, Mesenchymal Stem Cells (MSCs) are deemed to be the most potential adult stem cells for bone regeneration [2]. There are various tissues in the human body in which MSCs can be obtained, such as bone marrow, amniotic fluid, periosteum, adipose tissue, peripheral blood, and skin [3]. Bone Marrow-Derived MSCs (BM-MSCs), is the most widely used MSCs for bone regeneration due to its high osteogenic capacities compared to the others [4]. Nevertheless, BM-MSCs has some drawbacks, including the fact that bone marrow aspiration is a relatively invasive procedure. Therefore, the major challenge in cell therapy today is to find other sources of MSCs which impose minimal morbidity to the patients and yield a large number of quality stem cells.

Human Umbilical Cord is a source of numerous stem cells, which comprises of epithelial and stromal cells. The previous studies have proven that Human Umbilical Cord-Derived MSCs (hUCMSCs) was able to differentiate into three forms of germ layers, and has the anti-inflammatory effects as well as the low immunogenicity, hence avoiding the immune system and tissue rejection [5]. In its clinical application, hUCMSCs has a few advantages over the autogenous BM-MSCs, that is, its procurement procedures pose no morbidity, and there were unlimited amount of stem cells available due to its shorter expansion and doubling time than other adult stem cells [6]. In addition, the isolation of hUCMSCs does not kill the embryo, unlike embryonic stem cells. Hence, there are less ethical and legal issues involved [7].

The main constituent of the hydrogels developed is gelatin, according to biocompatibilty that gelatin is a non-toxic, biodegradable and water-soluble protein derived from collagen, with the latter being a major component of mesenchymal tissue extracellular matrix (ECM). Gelatin retains informational signals including an arginine-glycine-aspartic acid sequence, which promotes cell adhesion, proliferation and stem cell differentiation [8]. In a recent study, a group has demonstrated the use of a gelatin-coated flask for the successful generation of mesenchymal stem cells from either bone marrow stem cells or non amniotic placenta stem cells [9].

To provide potential clinical uses of hUCMSCs in bone tissue engineering, an effective hUCMSCs and its bone regeneration potential should be analysed and confirmed in vitro. This study aims to establish: 1) hUCMSCs phenotypic characterization. 2) The in vitro osteogenic differentiation potential of hUCMSCs. 3) The cytotoxicity of gelatin solvent to hUCMSCs using 3-[4,5-dimethylthiazol-2-yl]-2,5-diphenyl tetrazolium bromide (MTT) assay.

\section{Material and Methods}

\subsection{Collection and Preparation of Human Umbilical Cord}

Cesarean section was performed in the Central Operating Theatre of Dr. Soetomo General Hospital Surabaya on Healthy full term neonate. After the baby delivered, umbilical cord was cut out, the placenta together with the remaining umbilical cord was evacuated and placed in the sterile kidney-shape stainless steel receptacle. About $10 \mathrm{~cm}$ length of umbilical cord was obtained and washed in Phosphate Buffer Saline (PBS) three times to remove the excess blood and blood clot. Next, umbilical cord was soaked in Ringer Lactate solution containing $2.5 \mu \mathrm{g} / \mathrm{mL}$ gentamycin and $1000 \mathrm{U} / \mathrm{mL}$ amphotericin B for 20 minutes. The medical ethical review board of Dr. Soetomo General Hospital has already approved this protocol. 


\subsection{Isolation and Culture of hUCMSC}

Umbilical cord was dissected into small pieces about $1 \mathrm{~cm}$ and the umbilical arteries, veins and adventitia were removed to obtain the Wharton's Jelly. Wharton's Jelly was minced up with knife into fine pieces of $1 \mathrm{~mm}^{3}$ and was used to isolate and culture primary hUCMSCs.

Wharton's Jelly was transferred to $0.25 \%$ trypsin and digested in $37^{\circ} \mathrm{C}$ for 40 minutes, then centrifuged, have the supernatant removed, this processed was then repeated twice. The crushed and digested sample, then subjected to PBS containing $0.75 \mathrm{mg} / \mathrm{ml}$ collagenase IV (Sigma-Aldrich, St. Louis, MO, USA) and $0.075 \mathrm{mg} / \mathrm{mL}$ DNase I (Takara Bio, Shiga, Japan), incubated at $37^{\circ} \mathrm{C}$ for 60 minutes. This was followed by filtration with cell strainer and pellet collection upon centrifugation for 10 minutes to finally obtain the cells.

The single cells collected were then cultured on collagen-coated dishes using alpha modification of minimum essential medium eagle ( $\alpha$-MEM) (Gibco BRL, Gaithersburg, MD, USA), supplemented with human leukemia inhibitory factor $(10 \mathrm{ng} / \mathrm{mL}$ ) and fetal bovine serum (FBS) (Gibco BRL). Primary cells growth was observed under the microscope. The timing of cell confluence was recorded. The medium was changed once every three days. When the confluence reached $80 \%$, the cell splitting was done using trypsin. Half to two thirds of the cells were then re-plated onto a new dish of the same medium. The isolation procedure used was according to the Laboratory of Stem Cell, Institute of Tropical Disease, Airlangga University protocol.

\subsection{The hUCMSCs Phenotypic Characterization}

\subsubsection{Immunocytochemistry}

Cultured hUCMSCs were plated onto coverslips. The cells were rinsed five times with PBS tween. 20, drying and fixed with formaldehyde $10 \%$ for 15 minutes, and then they washed four times with PBS tween.20 and let to dry for few minutes. The cells were blocked by bovine serum albumin (BSA) for 30 minutes, incubated with fluorescein isiothiocyanate (FITC)-labeled monoclonal antibody anti-human Cellular Differentiation (CD)105 and CD45 for 60 minutes. Cells were rinsed with PBS twice and ready for analysis. Immunostained cells with CD105 and CD45 expression were analyzed using fluorescence microscope.

\subsubsection{Flow Cytometry}

Cultured hUCMSCs were trypsinized and suspended in $\alpha$-MEM, washed with PBS, and fixated in formaldehyde $10 \%$ for 10 minutes, and finally were closed in $10 \%$ BSA for one hour. Cells were incubated using Human MSCs Analysis Kit (BD Stemflow ${ }^{\mathrm{TM}}$, BD Biosciences) with primary antibodies mouse anti-human CD73, CD90, CD105, and negative cocktail containing CD45, CD34, CD19, CD11b, HLA-DR for 40 minutes. The unbound antibodies, then removed by washing with PBS. The bound primary antibodies were labelled using FITC-conjugated anti-mouse antibody by incubation for 30 minutes. The cells were then visualized and analyzed using FACS Calibur flow cytometer (BD Biosciences, Franklin Lakes, NJ, USA.

\subsection{In Vitro Osteogenic Differentiation Studies}

Cultured hUCMSCs at passage 8 were detached using $2 \times$ trypsin solution and seeded into 24-well microplate containing osteogenic medium, which composed of $\alpha$-MEM medium supplemented containing $50 \mu \mathrm{M}$ ascorbate phosphate, $10 \mu \mathrm{M}$ glycerol phosphate and $0.1 \mu \mathrm{M}$ dexamethasone, for osteogenic study.

The control group made of the same cells seeded into the same petri dish containing normal medium $(\alpha-$ MEM). Both of the medium were changed every 3 days. The cells were cultured in the osteogenic medium for 21 days to induce osteoblastic differentiation. After complete differentiation had been established morphologicaly, the cells were used for histochemical staining and immunohistochemistry studies. Cell fixation was performed by first detaching the cells from the petri dish of both media, followed by seeding into 24-well plate containing $10 \%$ formaldehyde. The presence of calcium deposits in the cultures was determined using Alizarin red stain according to a standard protocol to evaluate osteogenesis.

\subsection{Gelatin Solvent Mixture Procedure}

The next step was gelatin solvent preparation, Gelatin purchased from (Rousselot, VION company, Guangdong, China) was used in this research. $2 \%$ Gelatin was dissolved in a solution of $0.15 \mathrm{M}$ sodium chloride and $25 \mathrm{~m}$ MHEPES buffer at $\mathrm{pH} 7.0$ and sterilized by autoclaving. 


\subsection{Measuring Gelatin Solvent and $\alpha$-MEM Cytotoxicity to hUCMSCs}

MTT colorimetric assay was done in order to measure cytotoxicity of the gelatin solvent to the stem cells. Three sample groups were prepared: 1) Human Umbilical Cord Mesenchymal Stem Cells with gelatin solvent only; 2) Human Umbilical Cord Mesenchymal Stem Cells with gelatin solvent and $\alpha$-MEM medium; 3) Control group. Each group were prepared in a 96-well plates containing a final volume of $100 \mu \mathrm{l}$ per well for three times. After incubation, $10 \mu \mathrm{l}$ of MTT reagent was added to each well. The well then incubated for 4 hours at $37^{\circ} \mathrm{C} .100 \mu \mathrm{l}$ of Solubilization solution was added to each well with mixing to ensure complete solubilization. Viable cells would convert MTT into purple-coloured formazan crystals. The quantity of formazan is measured by recording changes in optical density at a wavelength using spectrophotometer.

\section{Result}

\subsection{Isolation and Culture of hUCMSCs}

After observation of growth for the first 24 hours, most of the cells were round or oval in shape. After 24 hours, the cells became adhere to the plate, and spindle in shape. After 3 days, the cells grew to a confluence of $90 \%$ and ware arranged in swirling pattern (Figure 1). Before the cells were used for subsequent examination, cell splitting procedure was performed 6 - 8 times.

\subsection{The hUCMSCs Phenotypic Characterization}

\subsubsection{Immunocytochemistry}

The expression of cell-surface antigens by Immunocytochemistry (ICC) and flow cytometry were evaluated in different passages. Immunocytochemistry showed that hUCMSCs expressed strong positive for CD45 and weak positive for CD105 at passage 6 (Figure 2).

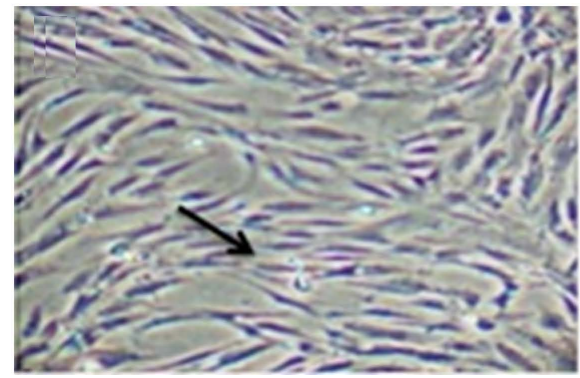

(a)

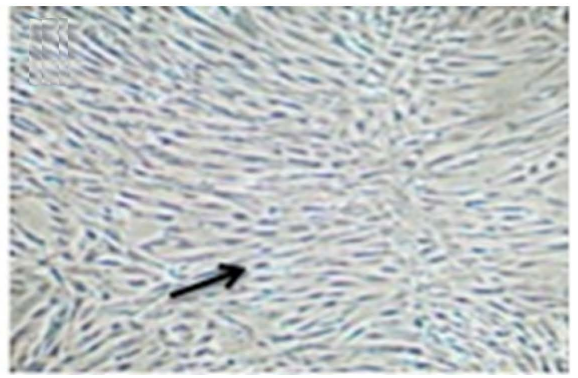

(b)

Figure 1. Culture of hUCMSCs. On the first 24 hours the cells were mostly round or oval in shape (a); after 3 days the majority of the cells were adherent to the plate, showing spindle-shape or fibroblast-like cell morphology with swirling-pattern colonies, and has reached confluence (b) (inverted microscope, 200× magnification).

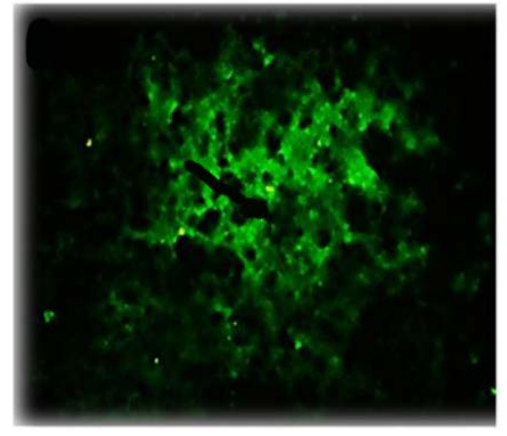

(a)

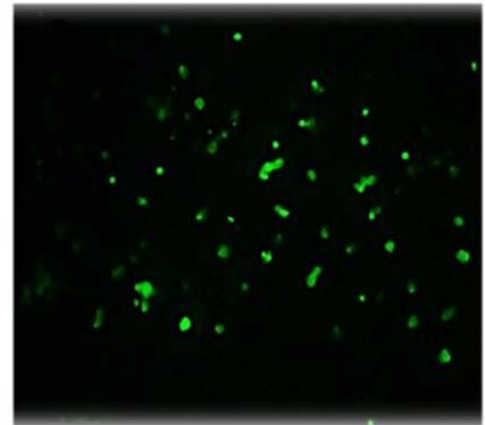

(b)

Figure 2. The immunocytochemistry result showed (a) that hUCMSC strongly expressed CD45 and (b) only weak expression of CD105 (immunofluorescence microscope, 100× magnification). 


\subsubsection{Flow Cytometry}

In flow cytometry, we did characterization at passage 8. The result showed that CD73 was the most significant and abundant surface receptors (CD73 ${ }^{+} \mathrm{CD} 105^{-}$in $95.55 \%$ subpopulation). Other MSCs markers showed smaller number of hUCMSCs subpopulation, such as $\mathrm{CD}^{+} 0^{+}$negPE $\mathrm{P}^{-}$(19.67\%) with only minimal subpopulation expressed $\mathrm{CD} 105$ (CD105 ${ }^{+}$negPE ${ }^{-}$in $0.19 \%$ subpopulation) and negative $\mathrm{PE}$ markers that containing negative MSC cocktail (CD45, CD34, CD11b, CD19, HLA-DR) (Figure 3).

\subsection{In Vitro Osteogenic Differentiation Studies}

After 21 days, majority of the cells in osteogenic medium were stained bright red with Alizarin Red, while only small quantity of cells in normal medium stained positively with Alizarin Red (Figure 4).

\subsection{Measuring Gelatin Solvent and $\alpha$-MEM Cytotoxicity to hUCMSCs}

Viable cells with active metabolism was able to change into purple coloured formazan crystals in MTT assay (Figure 5), whereas those which died lost its ability to do so. The quantity of formazan is measured by recording changes in optical density at a wavelength using spectrophotometer.

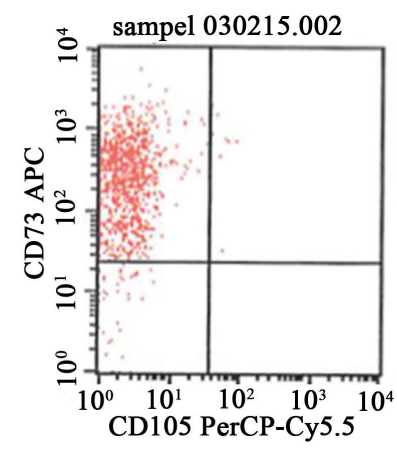

(a)

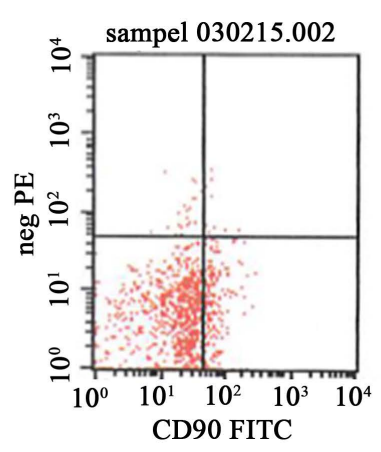

(b)

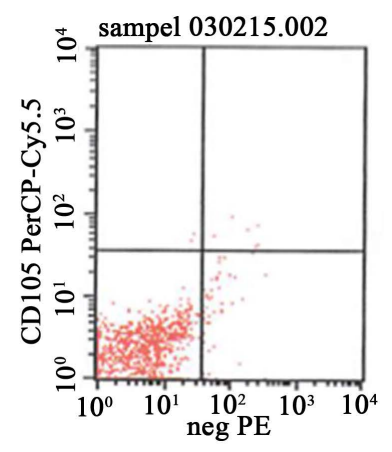

(c)

Figure 3. The flow cytometry result (a) showed that majority hUCMSCs subpopulation expressed CD73 and (b) with smaller subpopulation expressed CD90 and (c) only minimal number expressed CD105 and negative PE (containing negative MSC cocktail: CD45, CD34, CD11b, CD19, HLA-DR).

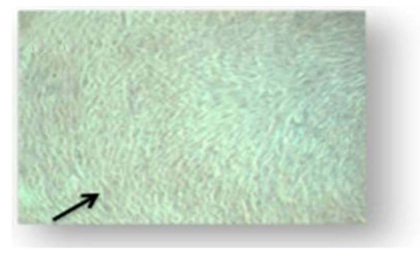

(a)

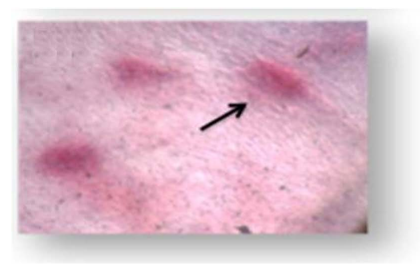

(c)

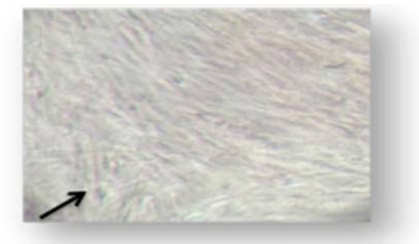

(b)

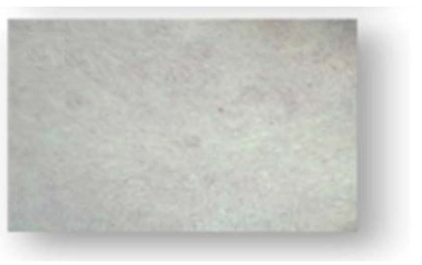

(d)

Figure 4. Microscopic feature of osteogenically-induced cell culture before and after staining with Alizarin Red. (a) hUCMSCs culture in osteogenic medium after 21 days showing morphology of round or oval-shaped cells and (b) in normal medium the cells showing features of MSCs; (c) Upon staining with Alizarin Red the osteogenic medium exhibited large clusters with bright red appearance indicating the presence of extracellular mineral deposit (d) while the normal medium showed no color changes detected (Alizarin Red staining, counter-stained with Mayer's hematoxylin, 200× magnification). 


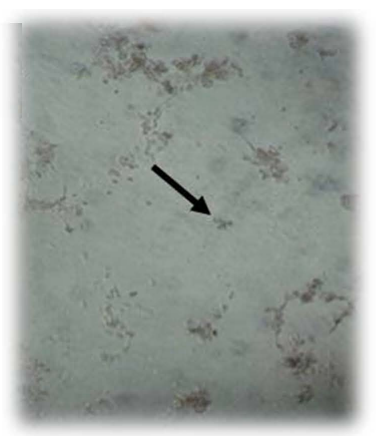

(a)

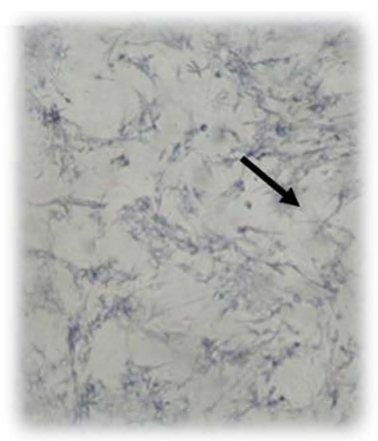

(b)

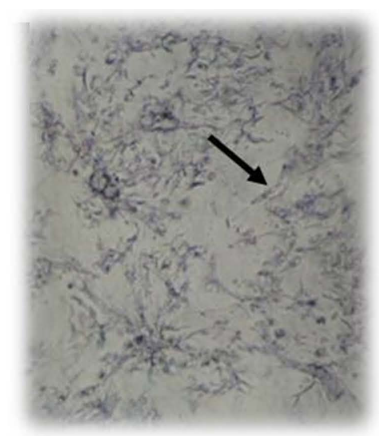

(c)

Figure 5. Pictures showing (a) Human Umbilical Cord Mesenchymal Stem Cells on MTT Assay on the control group; (b) Human Umbilical Cord Mesenchymal Stem Cells with gelatin solvent only and (c) Human Umbilical Cord Mesenchymal Stem Cells with gelatin solvent and $\alpha$-MEM).

For the purpose of this study, the cellular environment in which there were more than 50\% viable cells can be considered as safe and non-toxic. Based on the result shown, there are only $27.21 \%$ of the hUCMSCs are viable in gelatin solvent only. However, 53.98\% of the hUCMSCs are viable in gelatin solvent and $\alpha$ modification of MEM medium and can be considered non-toxic to hUCMSCs in vitro.

\section{Discussion}

In this study, the umbilical cord collections were all done by cesarean section under highly aseptic condition with purpose to prevents contamination effectively. It was done because procurement of umbilical cord in vaginal delivery, although much easier to perform, involves high incidence of fungalcontamination of the sample collected, and therefore not recommended. Study from Kamadjaja showed that all of the stem cell samples from amniotic membrane were contaminated with fungus even though performed in aseptic condition of vaginal delivery. Even a slight contamination of the sample, may eventually result in fungal overgrowing in the cell culture, rendering the sample no longer viable for the subsequent experiment [10].

The isolation protocol were using the Laboratory of Stem Cell, Institute of Tropical Disease, Airlangga University protocol and adopted from modified Kamadjaja study [10]. Since the method has been successfully used in this study, we made a slight modification on the medium used and has proven successful in isolating hUCMSCs. The features of the isolated cells were consistent with the characteristic morphology of MSCs shown by many studies in the literature. They were spindle-shaped fibroblast-like cells adhered to the petri dish. When the cell confluence was achieved, they acquired a swirling pattern [11]. This shows that the isolation method used in this study is also effective to isolate hUCMSCs, and is therefore recommended.

An interesting result obtained in characterization of our hUCMSCs. We did a characterization using immunocytochemistry and flow cytometry study on different passage. In immunocytochemistry study, CD45 was strongly expressed with weak expression of CD105 on passage 6. On contrary, in flow cytometry study that was done on passage 8, we found majority hUCMSCs subpopulation that expressed CD73, smaller subpopulation expressed CD90 with minimal number of cells that expressed CD105 and hematopoetic markers (CD45, CD34, CD11b, CD19, HLA-DR). The result on our study was similiar with Rammal study on Wharton Jelly MSC characterization. They found positive CD73, CD90 and CD105 expression as a MSC markers and negative CD34 and CD45 expression of hematopoietic markers [12]. Other studies also showed that positive expression of CD73, CD90 and CD105 were found as a characteristics of mesenchymal marker, and the the other criteria for MSC characterization was only less than 2\% of the MSC population will expressed CD34, CD45, CD11b, CD14, CD79, CD19 and HLA class II [6] [13] [14].

Additionally, the expression of CD73 was significantly levelled up as compared with the other two. Chatzistamatiou study also showed similar result with CD73 as the most expressed marker in Wharton's Jelly MSC [14]. As human amniotic MSCs also expresses the same set of markers of CD73, CD90, and CD105 [11], the significant expression of CD73 in human umbilical cord MSCs could be used as the distinguishing marker between the two. However, the exact quantitative difference between the two MSCs is out of the scope of this study. Hence, it could be further investigated in future studies. 
Our study showed positive but in smaller population of hUCMSCs that expressed CD90. Similiar with Rammal study, positive CD90 expression was found in Wharton's jelly mesenchymal stem cell [12]. Other research by Wiesmann, showing that under mechanical stimulation, CD90 was expressed during proliferation but will eventually declines (while there was increase of collagen I and osteonectin) as the cells mature towards osteoblast-like cell [15].

There were minimal subpopulation expressing CD105, this condition happened because the majority of Umbilical Cord membrane obtained in this research were possibly the ones with CD105 negative subpopulation, and may well be representing the actual condition of umbilical cord membrane subpopulation. Despite the low count of CD105, research by Levi, showed that CD105 depletion enhanced human adipose-derived stromal cell osteogenesis [16]. Similiar with this study, Leyva-Leyva observed that $82 \%$ of the cells population shared CD73 and CD44 antigens, but only $0.08 \%$ expressed CD105 on their surface. Cell passage can also significantly influence MSC marker expression. CD105 expression reached its maximum (10\%) after the first and second passages, but after the third and fourth passages, the expression fell down to 2\%. This explain the weak CD105 expression in $6^{\text {th }}$ cell passage during ICC and mostly negative cell subpopulation in 8th passage during flow cytometry examination [17]. On contrary with our study, research by Rantam showed that MSCs derived from rat bone marrow has $89.1 \%$ expression with flowcytometry [18].

There was an increase of CD45 on ICC study and negative expression on flow cytometry. One criteria of MSC characterization was a negative expression of CD45 as a hematopoetic marker. This condition happened because in ICC study, CD45 increase was counted with (qualitative) staining, insted of more accurate (quantitative) flow cytometry, therefore we concluded that immunocytochemistry study was not accurately depicting the actual CD45 level. Some diadvantage of ICC examination were potential of cross-reactivity when using multiple antibodies and potential of autofluorescence. Other possible explanation was due to isolation procedure, the MSCs colonies may not be purely those of isolated MSCs and not completely separated the hematopoeitic stem cells, depicting the increase of CD45 level [13] [14].

Alizarin Red staining was performed to detect osteoblastic differentiation in cell cultures by identifying the presence of extracellular calcium deposits. Stained osteoblasts will appear bright red in colour whereas undifferentiated MSCs do not [19]. This method is highly effective yet simple. In this study, the osteogenic-induced culture showed a significant amount of red-stained cell clusters, indicating that many of the hUCMSCs collected were differentiated towards osteoblast lineage in osteogenic environment. It showed that the osteogenic medium used in the study was effective. The result of this study were consisent with other studies that showed which under certain induction conditions, stem cells isolated from the human umbilical cord exhibit multilineage differentiation, one of which was toward osteoblasts [6] [20].

Ninety-five percents of $\mathrm{CD} 3^{+} \mathrm{CD} 105^{-}$hUCMSCs subpopulation in this study could be one of the osteogenic differentiation factor in this study. Leyva-Leyva study showed dissimilar osteoblastic differentiation potential between $\mathrm{CD}_{105^{+}}$and $\mathrm{CD}_{105^{-}}$[17]. Other study by Kmiecik showed a similiar subpopulation showed higher bone matrix mineralization, ostopontin and two other marker of mineralization than MSCs that expressed CD $44^{+} \mathrm{CD} 3^{+} \mathrm{CD}_{105^{+}}$[21]. Levi study also showed $\mathrm{CD} 105^{\text {low }}$ cells demonstrated significantly enhanced in vitro osteogenic differentiation and in vivo bone regeneration when compared with CD105 ${ }^{\text {high }}$ cells [16]. The quantification of bone matrix mineralization was clearly higher for the $\mathrm{CD}_{105^{-}}$than $\mathrm{CD}_{105^{+}}$subpopulations $^{-}$ [17]. This fact is good to emphasize the isolation of this specific subpopulation MSC for bone tissue engineering.

Moreover, more than half of the cultured cells were viable in gelatin and with $\alpha$ modification of MEM medium. This means that a combination of gelatin solvent and $\alpha$ modification of MEM medium is safe and nontoxic to the hUCMSCs in vitro. This result was consistent with previous study which showed that human cells, though not necessarily hUCMSCs, could survive in gelatin environment [22] [23]. Therefore, by extension, the study suggests the use of a combination of gelatin solvent and $\alpha$-MEM medium as a safe and fairly effective medium of delivery of hUCMSCs in vivo.

\section{Conclusion}

In conclusion,the study has revealed that the MSCs isolated from the human umbilical cord (hUCMSCs) had the capacity for in vitro osteogenesis, listing them as one of the potential allogeneic MSCs for bonecell therapy. This study also suggests the use of a combination of gelatin solvent and $\alpha$-MEM medium as a safe and fairly effective medium of delivery of hUCMSCsin vitro for bone regeneration.However, in vivo studies and further 
research should be done to investigate its clinical application potential in bone tissue engineering.

\section{References}

[1] Holy, C., Volenec, F., Geesin, J. and Bruder, S. (2007) Principles of Tissue Engineering. 3rd Edition, Elsevier Academic Press, Burlington.

[2] Undale, A., Westendorf, J., Yaszemski, M. and Khosia, S. (2009) Mesenchymal Stem Cells for Bone Repair and Metabolic Bone Diseases. Mayo clinic proceedings, 84, 893-902. http://dx.doi.org/10.4065/84.10.893

[3] Sudo, K., Kanno, M., Miharada, K., Ogawa, S., Hiroyama, T., Saijo, K. and Nakamura, Y. (2007) Mesenchymal Progenitors Able to Differentiate into Osteogenic, Chondrogenic and/or Adipogenic Cells in Vitro Are Present in Most Primary Fibroblast-Like Cell Population. Stem Cells, 25, 1610-1617. http://dx.doi.org/10.1634/stemcells.2006-0504

[4] Augello, A., Kurth, T. and de Bari, C. (2010) Mesenchymal Stem Cells: A Perspective from in Vitro Cultures to in Vivo Migration and Niches. European Cells and Materials, 20, 121-133.

[5] Ilancheran, S., Michalska, A., Peh, G., Wallace, E., Pera, M. and Manuelpillai, U. (2007) Stem Cells Derived from Human Fetal Membranes Display Multilineage Differentiation Potential. Biology of Reproduction, 77, 577-588. http://dx.doi.org/10.1095/biolreprod.106.055244

[6] Wang, L., Ott, L., Seshareddy, K., Weiss, M. and Detamore, M. (2011) Musculoskeletal Tissue Engineering with Human Umbilical Cord Mesenchymal Stromal Cells. Regenerative Medicine, 6, 95-109. http://dx.doi.org/10.2217/rme.10.98

[7] Lidenmair, A., Hatlapatka, T., Kollwig, G., Hennerbichler, S., Gabriel, C., Wolbank, S., Redl, H. and Kasper, C. (2012) Mesenchymal Stem or Stromal Cells from Amnion and Umbilical Cord Tissue and Their Potential for Clinical Applications. Cells, 1, 1061-1088. http://dx.doi.org/10.3390/cells1041061

[8] Salamon, A., van Vlierberghe, S., van Nieuenhove, I., Baudisch, F., Graulus, G., Benecke, V., Alberti, K., Meumann, H., Rychly, J., Martins, J., Dubruel, P. and Peters, K. (2014) Gelatin-Based Hydrogels Promote Chondrogenic Differentiation of Human Adipose Tissue-Derived Mesenchymal Stem Cells in Vitro. Materials, 7, 1342-1359. http://dx.doi.org/10.3390/ma7021342

[9] Li, C., Liu, I., Tsao, C. and Chan, V. (2014) Neuronal Differentiation of Human Placenta—Derived Multi-Potent Stem Cells Enhanced by Cell Body Oscillation on Gelatin Hydrogel. Journal of Bioactive and Compatible Polymers, 29, 529-544. http://dx.doi.org/10.1177/0883911514553903

[10] Kamadjaja, D., Purwati, Rantam, F., Ferdiansyah and Pramono, C. (2014) The Osteogenic Capacity of Human Amniotic Membrane Mesenchymal Stem Cell (hAMSC) and Potential Application in Maxillofascial Bone Reconstruction in Vitro Study. Journal of Biomedical Science and Engineering, 7, 497-503. http://dx.doi.org/10.4236/jbise.2014.78051

[11] Huo, S.Z., Shi, P. and Pang, X.N. (2010) Culture and Identification of Human Amniotic Mesenchymal Stem Cells. Chinese Medical Sciences Journal, 25, 211-214. http://dx.doi.org/10.1016/S1001-9294(11)60004-7

[12] Rammal, H., Beroud, J., Gentils, M., Labrude, P., Menu, P., Kerdjoudj, H. and Velot, E. (2013) Reversing Charges or How to Improve Wharton's Jelly Mesenchymal Stem Cells Culture on Polyelectrolyte Multilayer Films. Biomedical Materials and Engineering, 23, 299-309. http://dx.doi.org/10.3233/BME-130754

[13] Wang, H., Hung, S., Peng, S., Huang, C., Wei, H., Guo, Y., Fu, Y., Lai, M. and Chen, C. (2004) Mesenchymal Stem Cells in the Wharton's Jelly of the Human Umbilical Cord. Stem Cells, 22, 1330-1337. http://dx.doi.org/10.1634/stemcells.2004-0013

[14] Chatzistamatiou, T., Papassavas, A., Michalopoulos, E., Gamaloutsos, C., Mallis, P., Gontika, I., Panagouli, E., Koussoulakos, S. and Stavropoulos-Giokas, C. (2014) Optimizing Isolation Culture and Freezing Methods to Preserve Wharton’s Jelly's Mesenchymal Stem Cell (MSC) Properties: An MSC Banking Protocol Validation for the Hellenic Cord Blood Bank. Transfusion, 54, 3108-3120. http://dx.doi.org/10.1111/trf.12743

[15] Wiesmann, A., Buhring, H.J., Mentrup, C. and Weismann, H.P. (2006) Decreased CD90 Expression in Human Mesenchymal Stem Cells by Applying Mechanical Stimulation. Head and face Medicine, 2, 8. http://dx.doi.org/10.1186/1746-160X-2-8

[16] Levi, B., Wan, D., Glotzbach, J., Hyun, J., Januszyk, M., Montoro, D., Sorkin, M., James, A., Nelson, E., Li, S., Quarto, N., Lee, M., Gurtner, G. and Longaker, M. (2011) CD105 Protein Depletion Enhances Human Adipose-Derived Stromal Cell Osteogenesis through Reduction of Transforming Growth Factor B1 (TGF-B1) Signaling. The Journal of Biological Chemistry, 286, 39497-39509. http://dx.doi.org/10.1074/jbc.M111.256529

[17] Leyva-Leyva, M., Barrera, L., Lopez-Camarillo, C., Arriaga-Pizano, L., Orozco-Hoyuela, G., Carrillo-Casas, E.M., Calderon-Perez, J., Lopez-Dıa, A., Hernandez-Aguilar, F., Gonzalez-Ramırez, R., Kawa, S., Chimal-Monroy, J. and Fuentes-Mera, L. (2013) Characterization of Mesenchymal Stem Cell Subpopulation from Human Amniotic Membrane with Dissimilar Osteoblastic Potential. Stem Cells and Development, 22, 1275-1287.

http://dx.doi.org/10.1089/scd.2012.0359 
[18] Rantam, F.A., Purwati, Setiawan, B., Wibisono, S., Ferdiansyah., Wahyuhadi, J., Mouli, E., Utomo, D.N., Suroto, H. and Bumi, C. (2015) Induced Monocytes-Derived HSCs (CD34 ${ }^{+}$) with LPS Accelerated Homing Rat Bone Mar-RowMesenchymal Stem Cell (BM-MSCs, CD105) in Injured Pancreas. Journal Biomedical Science and Engineering, 8, 111. http://file.scirp.org/Html/3-9102133_56226.htm

[19] Gregory, C., Gunn, W., Peister, A. and Prockop, D. (2005) An Alizarin Red-Based Assay of Mineralization by Adherent Cells in Culture: Comparison with Cetylpyridinium Chloride Extraction. Analytical Biochemistry, 329, 77-84. http://dx.doi.org/10.1016/j.ab.2004.02.002

[20] Kouroupis, D., Churchman, S., English, A., Emery, P. and Giannoudis, P. (2013) Assessment of Umbilical Cord Tissue as a Source of Mesenchymal Stem Cell/Endothelial Cell Mixtures for Bone Regeneration. Regenerative Medicine, 8, 569-581. http://dx.doi.org/10.2217/rme.13.47

[21] Kmiecik, G., Spoldi, V., Silini, A. and Parolini, O. (2014) Current View on Osteogenic Differentiation Potential of Mesenchymal Stromal Cells Derived from Placental Tissues. Stem Cell Reviews and Report, 1-16.

[22] Li, M., Cui, T., Mills, D.K., Lyov, Y.M. and Mcshane, M.J. (2005) Comparison of Selective Attachment and Growth of Smooth Muscle Cells on Gelatin and Fibronectin Coated Micropatterns. Journal of Nanoscience and Nanotechnology, 5, 1809-1885. http://dx.doi.org/10.1166/jnn.2005.436

[23] Tzouanas, S.N., Ekenseair, A.K., Kasper, F.K. and Mikos, A.G. (2014) Mesenchymal Stem Cell and Gelatin Microparticle Encapsulation in Thermally and Chemically Gelling Injectable Hydrogels for Tissue Engineering. Journal of Biomedical Material Research Part A, 102, 1222-1230. http://dx.doi.org/10.1002/jbm.a.35093 